\title{
Multi-model inference using mixed effects from a linear regression based genetic algorithm
}

\author{
Koen Van der Borght ${ }^{1,2^{*}}$, Geert Verbeke ${ }^{2,3}$ and Herman van Vlijmen ${ }^{1}$
}

\begin{abstract}
Background: Different high-dimensional regression methodologies exist for the selection of variables to predict a continuous variable. To improve the variable selection in case clustered observations are present in the training data, an extension towards mixed-effects modeling (MM) is requested, but may not always be straightforward to implement.

In this article, we developed such a MM extension (GA-MM-MMI) for the automated variable selection by a linear regression based genetic algorithm (GA) using multi-model inference (MMI). We exemplify our approach by training a linear regression model for prediction of resistance to the integrase inhibitor Raltegravir (RAL) on a genotype-phenotype database, with many integrase mutations as candidate covariates. The genotype-phenotype pairs in this database were derived from a limited number of subjects, with presence of multiple data points from the same subject, and with an intra-class correlation of 0.92 .

Results: In generation of the RAL model, we took computational efficiency into account by optimizing the GA parameters one by one, and by using tournament selection. To derive the main GA parameters we used 3 times 5 -fold cross-validation. The number of integrase mutations to be used as covariates in the mixed effects models was 25 (chrom.size). A GA solution was found when $R_{M M}^{2}>0.95$ (goal.fitness). We tested three different MMI approaches to combine the results of $100 \mathrm{GA}$ solutions into one GA-MM-MMI model. When evaluating the GA-MM-MMI performance on two unseen data sets, a more parsimonious and interpretable model was found (GA-MM-MMI TOP18: mixed-effects model containing the 18 most prevalent mutations in the GA solutions, refitted on the training data) with better predictive accuracy $\left(\mathrm{R}^{2}\right)$ in comparison to GA-ordinary least squares (GA-OLS) and Least Absolute Shrinkage and Selection Operator (LASSO).

Conclusions: We have demonstrated improved performance when using GA-MM-MMI for selection of mutations on a genotype-phenotype data set. As we largely automated setting the GA parameters, the method should be applicable on similar datasets with clustered observations.
\end{abstract}

Keywords: Variable selection, Linear regression, Genetic algorithm, Mixed-effects model, Multi-model inference

\section{Background}

In recent studies, classical regression methods for prediction of a continuous variable from a large number of covariates have been extended for the training of a model when the data set is hierarchical in nature [1-4]. In this article we extend our genetic algorithm (GA) variable selection methodology in [5] to allow for clustering in the data. We compare the performance of multi-model inference (MMI)

\footnotetext{
*Correspondence: kvdborgh@its.jnj.com

${ }^{1}$ Janssen Infectious Diseases-Diagnostics BVBA, B-2340 Beerse, Belgium

${ }^{2}$ Interuniversity Institute for Biostatistics and statistical Bioinformatics,

Katholieke Universiteit Leuven, B-3000 Leuven, Belgium

Full list of author information is available at the end of the article
}

using restricted maximum likelihood (REML) mixed-effects modeling [6,7] (MM) with ordinary least squares regression [8] (OLS) and compare GA-MMI with the commonly used penalized regression method Least Absolute Shrinkage and Selection Operator [9] (LASSO). We also show how to optimally set the GA parameters.

As an example, the training of a linear regression model for prediction of Raltegravir (RAL) resistance ("phenotype") from mutations in the HIV integrase region ("genotype") is worked out. The data sets used for training and testing were described in more detail in [5]. The training set consisted of $n=991$ clonal genotype-phenotype measurements, from multiple clones derived from 153 clinical isolates 
(on average 5 à 6 clones per isolate) and repeated measurements (on average 3) from 28 site-directed mutants (in-vitro lab created clones with a designed mutational pattern), and the number of candidate mutations for selection was $p=322$. Two test sets were used: the first consisted of population data of 171 clinical isolates (test set 1), the second consisted of 67 integrase sitedirected mutants containing most of the known RAL resistance associated mutational patterns [10] (test set 2). As it was found in [5] that a second order model did not significantly outperform a first order model, we did not consider interaction terms.

The paper is organized as follows. We begin by recalling the Simple Genetic Algorithm for variable selection in OLS linear regression. Then, we introduce GA-MM as an extension for clustered data. Finally, we introduce MMI for estimation of the model parameters, combining the results from multiple GA-MM (or GA-OLS) runs, followed by a short section on how we applied the LASSO method for comparison. In the remainder of the paper, we illustrate our methodology on an example for the predictive modeling of RAL resistance. For this example, we describe in detail how we optimized the GA parameter settings, and we report the results of comparing GA-MMMMI with LASSO and GA-OLS-MMI. When nominating one 'best' model, from all models evaluated in the comparison, we chose the GA-MM-MMI TOP18 model as a sparse model with high biological relevance (17 out of 18 integrase mutations in this model have been confirmed to be associated with resistance [5]), and having better predictive accuracy than LASSO and GA-OLS-MMI models with equal number of mutations selected. Throughout the text of this article GA related terminology is written in italic.

\section{Methods \\ GA-OLS}

The Simple Genetic Algorithm, due to John Holland [11-15], is used to evaluate a set P of regression models $\mathrm{M}$ with $p_{\text {sel }}$ variables. In GA terminology: $\mathrm{P}$ is a population, and a model $\mathrm{M} \in \mathrm{P}$, is called an individual, the $p_{\text {sel }}$ model variables determine the individuals' chromosome. The number of models in a population, $|\mathrm{P}|$, is fixed, as well as the number of variables $p_{\text {sel }}$ in a model M. In GA terminology: $|\mathrm{P}|$ is called the population size (pop.size), and $p_{\text {sel }}$ is called the chromosome size (chrom.size). Thus, each regression model $M$ represents a candidate subset of $p_{\text {sel }}$ variables (in GA terminology variables are called genes), and a GA fitness function has to be defined to identify the better or 'more fit' individuals. In GA-OLS, we used the linear model $\mathrm{R}^{2}$ (OLS) goodness-of-fit statistic as fitness function. The better the model $M$ fits to the data, the higher $\mathrm{R}^{2}$ (with $0 \leq \mathrm{R}^{2} \leq 1$ ). Models with $\mathrm{R}^{2}>$ goal fitness are termed solutions to the optimization problem. A Darwinian evolution is applied to modify the population over several generations. The GA finds a solution using the search procedure as given in Table 1.

In step 3 of Table 1, the mutation genetic operator alters a gene (replacing it with another gene from the pool of candidate genes) in a chromosome with probability Pm. The crossover genetic operator re-combines the genotypes of two individuals. The probability of an individual to be selected for crossover is Pc. The key in the optimization is to keep a good balance between selective pressure (Table 1 step 2) and genetic diversity (Table 1 step 3). The GA run is completed when an individual is found with fitness $>$ goal fitness. When no solution is found within a maximum number of generations (max.generations), the GA run is halted. For step 2 of Table 1, we used tournament selection as detailed in Section II (Results and discussion). Also, elitism is used, meaning that the best chromosome (highest $R^{2}$ ) is passed through to the next generation, with a probability $P e$.

The running of the GA is done multiple times to generate a set $S$ of solutions. A ranking by importance can then be made for all variables based on their frequency in $S$.

\section{GA-MM}

Although OLS parameter estimates are known to be unbiased when neglecting the correlation structure [6], in this article we want to evaluate whether using a mixed model for the GA models, using a random subject effect in addition to the fixed effects (variables as in the OLS model), can improve the interpretability or performance of the final linear regression model, derived with MMI (next section).

The GA-MM methodology makes use of the Simple Genetic Algorithm (Table 1), completely analogous to GA-OLS, producing a ranking of variables by their frequency in a set $S$ of GA solutions. However, there is no single commonly used definition for the $\mathrm{R}^{2}$ statistic as is the case for OLS $[16,17]$. Several definitions have been suggested that all have different interpretations in the presence of correlated errors. Here, we used the marginal $R_{M M}^{2}$ definition from [18], quantifying the variance explained by the fixed effects. As new data will originate from other subjects than those used for the training of the model, the random effects cannot be used for prediction. In [1] it has also been described that conditional $\mathrm{R}^{2}$ (variance explained by the entire model, including the

\section{Table 1 Simple genetic algorithm}

\begin{tabular}{ll}
\hline Step & Description \\
1 & Initialize a random population of pop.size individuals, goto step 4. \\
2 & Select the more fit individuals to form a new population. \\
3 & Modify genetic material of the individuals in this new population \\
& by applying genetic operators: mutation and cross-over. \\
4 & Evaluate fitness of the population. If no solution found goto \\
& step 2, else end.
\end{tabular}


random effects) should not be used for fixed-effect variable selection. For us, the main motivation for using $R_{M M}^{2}$ was that the MM can be fitted using REML, resulting in better estimates for the variance components, needed in the estimation of the fixed effects, especially in models with many fixed effects [7].

In the example, for predicting the RAL phenotype $y$ from the integrase clonal genotype $x \in[0,1]^{p}$, the mixed model $M$ uses one random effect/ cluster factor $\alpha_{i}$ (clones are clustered per clinical isolate/ site-directed mutant):

$$
y_{i j}=\beta_{0}+\sum_{k=1}^{p} \beta_{k} x_{k i j}+\alpha_{i}+\varepsilon_{i j},
$$

with $\beta_{0}$ the intercept, and $y_{i j}$ the $\mathrm{j}$-th response of cluster $\mathrm{i}$,

$$
\alpha_{i} \sim N\left(0, \sigma_{\alpha}^{2}\right),
$$

and

$$
\begin{gathered}
\varepsilon_{i j} \sim N\left(0, \sigma_{\varepsilon}^{2}\right) \\
\text { If } x_{k} \notin M: \beta_{k} \equiv 0 .
\end{gathered}
$$

The marginal $R^{2}$ is calculated as:

$$
R_{M M}^{2}=\frac{\sigma_{f}^{2}}{\sigma_{f}^{2}+\sigma_{\alpha}^{2}+\sigma_{\varepsilon}^{2}},
$$

where $\sigma_{f}^{2}$ is the variance calculated from the fixed effects $\beta_{k}$ :

$$
\sigma_{f}^{2}=\operatorname{var}\left(\sum_{k=1}^{p} \beta_{k} x_{k i j}\right),
$$

$\sigma_{\alpha}^{2}$ is the between-cluster variance, and $\sigma_{\varepsilon}^{2}$ is the withincluster variance.

The intra-class correlation: $I C C=\frac{\sigma_{\alpha}^{2}}{\sigma_{\alpha}^{2}+\sigma_{\varepsilon}^{2}}$ for the model without fixed effects was 0.92, showing very strong within-cluster correlation, and suggesting that accounting for this correlation may improve the performance of our model.

\section{GA-MMI}

In $[19,20]$ it has been described that, when the number of samples in the training data is small, making inference from a single best model, e.g., produced with stepwise regression, leads to the inclusion of noise variables. Here, we used MMI to combine the information from the GA solutions into a final model for making predictions. As a GA run is stopped as soon as the goal fitness (calculated in section VI (Results and discussion)) is achieved (Table 1, step 4), GA solutions were 'equally fit'. Thus, we used equal weighting of the GA solutions in the MMI. In [6] it was shown that for stepwise regression using an information criterion for selection - as we used in [5] for deriving a consensus model from the GA ranking of variable frequencies - one should for MM use the biased ML estimators. An advantage of using MMI in combination with GA-MM is that REML can still be used. Thus, using MMI, we could make a fair comparison between GA-OLS and GA-MM.

For estimation of the parameters for the final model, we used the following three MMI approaches on the GA solutions:

1. Refitting for a TOP selection of the GA ranking: from the GA-ranking, the variables with highest frequencies were retained for the final model, which was then refitted using OLS/MM.

2. Averaging of parameter estimates $\hat{\beta}_{k}$ using all GA solutions $\left(\hat{\beta}_{k} \equiv 0\right.$, if $x_{k}$ not in GA solution) (MMI1):

$$
\bar{\beta}_{k}=\frac{\sum_{s=1}^{|S|} \hat{\beta}_{k s}}{|S|},
$$

with $|S|$ the number of GA solutions.

3. Averaging of parameter estimates $\hat{\beta}_{k}$ using GA solutions where $\hat{\beta}_{k} \neq 0$ (MMI2):

$$
\bar{\beta}_{k}=\frac{\sum_{s=1}^{|S|} \hat{\beta}_{k s}}{\left|\left\{M \in S \mid x_{k} \in M\right\}\right| .}
$$

For the model averaging in 2 and 3, parameters $\hat{\beta}_{k}$ were (re-)fitted using OLS/MM for all $m$ variables with presence at least once in a GA solution or for a TOP selection of variables in the GA ranking only.

\section{LASSO}

LASSO [9] is a regularization method that performs variable selection by constraining the size of the coefficients, also called shrinkage. By applying an L1 absolute value penalty, regression coefficients are 'shrunk' towards zero, forcing some of the regression coefficients to zero. Using the R package glmselect 1.9-3 [21], for the described example in this paper we performed variable selection using the LASSO technique on the clonal genotype-phenotype database returning a LASSO ranking of variables (solution path) as selected by decreasing the amount of penalty applied. Besides using the shrinkage coefficients for variable estimation (default LASSO) we also applied OLS and MM to the LASSO selected variables (post-LASSO [22]). 


\section{Results and discussion GA parameter settings}

We optimized the GA parameters one by one in the order $(\mathbf{I}->$ VI $)$ as described below, and taking computational efficiency into account (see Additional file 1). Tournament selection was used as selection method to form a new population of more fit individuals. GA parameters $P m$ and $P c$ were optimized together using a meta-GA. Pe and pop.size were fixed in advance and were not optimized. $\mathrm{Pe}$ was set to conserve the best chromosome in three consecutive generations, followed by a generation where the probability of keeping the best chromosome was set to $20 \%$. Pop.size was set equal to 20. To set the main GA parameters: max.generations, chrom.size, and num.runs we used cross-validation (Additional file 1 point 7).

For running the GA, we used the R package GALGO 1.0.11 [23]. After inspection of the $R_{C V}^{2}$ results, with exception of goal.fitness, we took the same optimized GA parameters values for GA-OLS and GA-MM (for the model comparison): pop.size $=20$, chrom.size $=25$, $P m=0.1, P c=0.6, P e=(1,1,1,0.2)$, max. generations $=500$, tournament.size $=10$, num.solutions $=100$, goal.fitness. ols $=0.957$, and goal.fitness. $m m=0.95$. In Additional file 2 is the $\mathrm{R}$ code we used to derive these settings and to run GA-MM-MMI.

\section{Meta-GA for selection of Pm and Pc}

For the meta-GA optimization of the parameters $P m$ and $P_{c}$ (Table 2), we used the $\mathrm{R}$ package gaoptim 1.0 with the default settings (except for pop.size meta $=20$, instead of 100 (default)) [24]. GA-OLS was used as the meta-GA fitness function returning the $\mathrm{R}^{2}$ from the best chromosome for the $(P m, P c)$ combinations. Different random numbers were generated for each of the GA-OLS runs, thus the same real-valued combination $(P m, P c)$ with multiple presence in the meta-GA population did not give the same fitness result. The fitness

Table 2 Meta-GA optimization of $P m$ and $P C$

\begin{tabular}{|c|c|c|c|}
\hline$G A$ & CHOSEN & PRE-SET & $\begin{array}{l}\text { BEING } \\
\text { OPTIMIZED }\end{array}$ \\
\hline \multirow[t]{5}{*}{ GA-OLS } & pop.size $=20$ & chrom.size $=15$ & $P m \in[0,1]$ \\
\hline & $P e=(1,1,1,0.2)$ & max generations $=100$ & $P C \in[0,1]$ \\
\hline & & num.runs $=1$ & \\
\hline & & goal.fitness $=1$ & \\
\hline & & tournament.size $=10$ & \\
\hline \multirow[t]{5}{*}{ metaGA } & pop.size $e_{\text {meta }}=20$ & & \\
\hline & num.generations $_{\text {meta }}=100$ & & \\
\hline & $P m_{\text {meta }}=0.01$ & & \\
\hline & $P C_{\text {meta }}=0.9$ & & \\
\hline & $P e_{\text {meta }}=0.4$ & & \\
\hline
\end{tabular}

landscape from 2000 (pop.size meta $\times$ num.generations meta $_{\text {) }}$ ) points is shown in Figure 1.

Crossover was a fairly weak genetic operator as can be seen from the red band in Figure 1. Oppositely, the mutation genetic operator was a strong operator and was best taken in the range [0.1, 0.4]. The meta-GA converged at $(P m, P c)=(0.258,0.372)$. For further evaluation in Section II, we also selected $(0.1,0.6)$ and $(0.2,0.6)$ located in the largest dark red area in Figure $1\left(R^{2}>0.91\right)$.

\section{Tournament selection}

Tournament selection [15,25] is a selection method to bias the selection towards the more fit individuals. Pop.size tournaments are organized with $\mathrm{k}$ randomly selected chromosomes. The winner of a tournament is the chromosome with the best fitness (highest $\mathrm{R}^{2}$ ). The pop.size tournament winners become the new population. Selection pressure, the degree to which better individuals are favoured, is increased when the tournament size is increased, as the winner from a large tournament will, on average, have a higher fitness than the winner of a small tournament.

In the optimization (Table 3), all tournament sizes $1 \leq \mathrm{k} \leq$ pop.size were considered. From section I, we selected the following $(P m, P c)$ combinations for evaluation: $(0.1,0.6),(0.2,0.6)$, and $(0.258,0.372)$. We also considered $(0.05,0.7)$, the $(P m, P c)$ combination used in [5]. From Figure 2 , to improve the $\mathrm{R}^{2}(\mathrm{OLS})$ performance the tournament.size $k$ should be taken $>2$. We chose to continue to use $k=10$ (as pre-set in section I). Slightly better $\mathrm{R}^{2}$ performance was seen for the $(P m, P c)$ combinations $(0.1,0.6)$ and $(0.2,0.6)$. The former was chosen for reasons of computational efficiency.

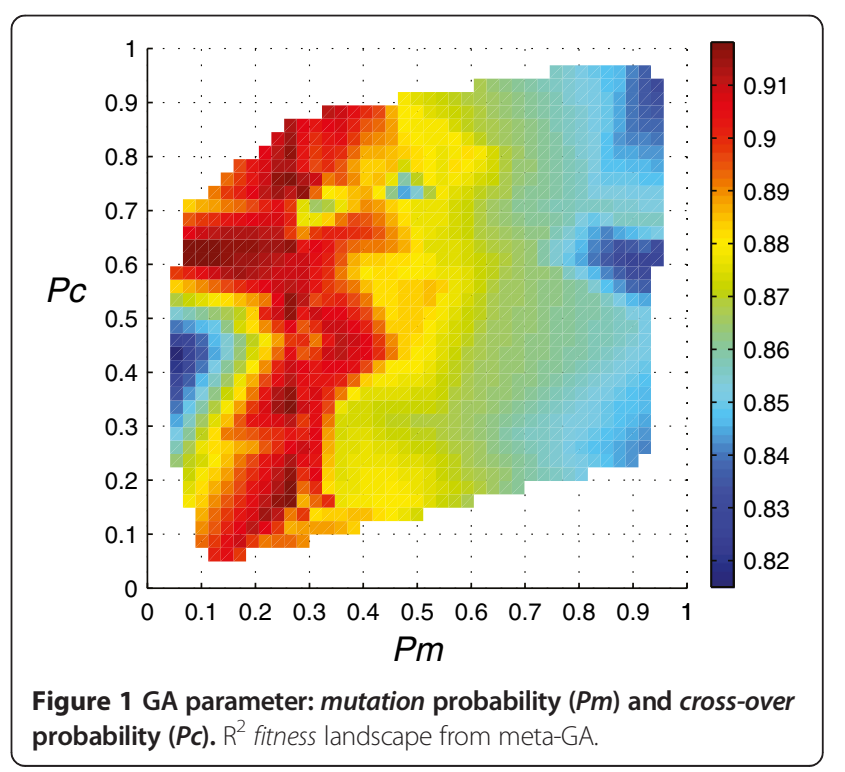


Table 3 GA parameter settings to evaluate tournament.size and $(P m, P c)$

\begin{tabular}{llll}
\hline GA & CHOSEN & PRE-SET & BEING OPTIMIZED \\
\hline GA-OLS & pop.size $=20$ & chrom.size $=15$ & tournament.size $\in\{1, \ldots, 20\}$ \\
& $P e=(1,1,1,0.2)$ & max.generations $=100$ & $(P m, P C) \in\{(0.1,0.6) ;(0.2,0.6) ;$ \\
& & num.runs $=10$ & $(0.258,0.372) ;$ \\
& & goal.fitness $=1$ & $(0.05,0.7)\}$ \\
\hline
\end{tabular}

\section{Maximum number of generations}

In Table 4, the GA settings for evaluating max.generations are summarized. Evaluation was done for both GA-OLS and GA-MM, calculating $R_{C V}^{2}$ as the mean from 3 repetitions of 5-fold cross-validation: $R_{C V}^{2}=\sum_{i=1}^{3} R_{C V, i}^{2} / 3$ where $R_{C V, i}^{2}$ was calculated as the correlation between the phenotype measurements of all observations in the database (contained exactly once in test set $T_{i j}, j=1 \ldots 5$ ) and their mean prediction (MMI1) of the 10 best chromosomes from GA-OLS/GA-MM (trained on train set $T R_{i j}$ containing $4 / 5$ of the subjects).

From Figure 3, it can be seen that, while the improvement in $\mathrm{R}_{\mathrm{CV}}^{2}$ when increasing max.generations from 100 to 300 was larger for GA-MM than for GA-OLS, the $\mathrm{R}_{\mathrm{CV}}^{2}$ performance for GA-MM was found to be lower than for GA-OLS. Stabilization of $\mathrm{R}_{\mathrm{CV}}^{2}$ was seen for both GA-OLS and GA-MM for num.generations $\geq 400$. We chose max.generations $=400$ to be used further in the sections IV and V. Note that for the pre-set goal fitness $=1$, max.generations was the number of generations always executed. For the model comparison, with the goal fitness calculated (Section VI), we set max.generations $=500$ for both GA-OLS and GA-MM.

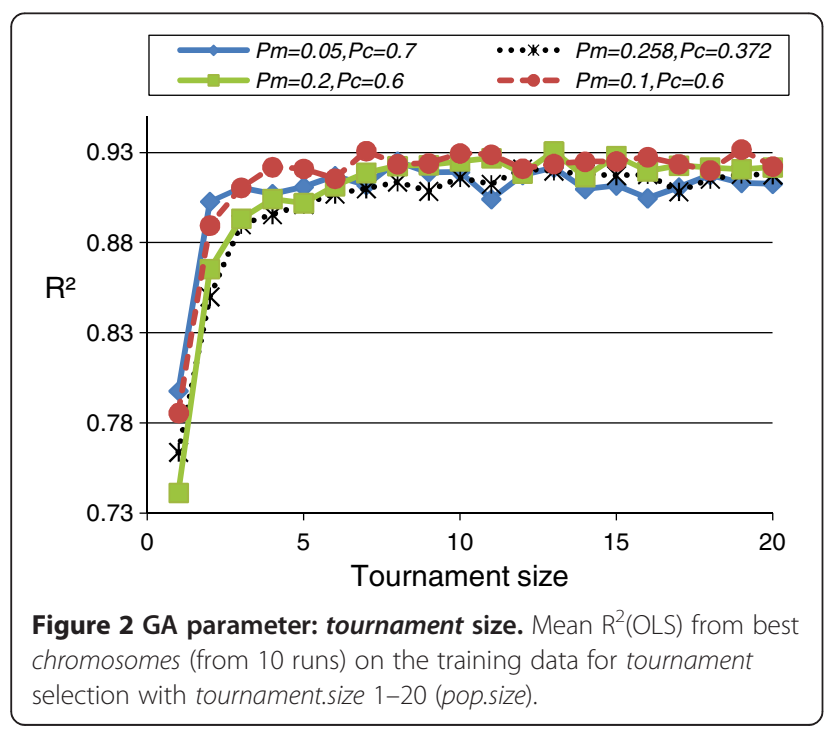

Table 4 GA parameter settings to evaluate max.generations

\begin{tabular}{llll}
\hline GA & CHOSEN & PRE-SET & BEING OPTIMIZED \\
\hline GA-OLS & pop.size $=20$ & chrom.size $=15$ & max.generations $\in$ \\
GA-MM & $P e=(1,1,1,0.2)$ & num.runs $=10$ & $\{100,200,300,400,500\}$ \\
& $P m=0.1$ & goal.fitness $=1$ & \\
& $P C=0.6$ & & \\
& tournament.size $=10$ & & \\
\end{tabular}

\section{Chromosome size}

In Table 5 the GA settings for evaluating chrom.size are presented. Analogously as for num.generations, evaluation was done for GA-OLS as well as GA-MM, using $3 \times 5$-fold cross-validation (see section III).

The $\mathrm{R}_{\mathrm{CV}}^{2}$ performance is shown in Figure 4. Stabilization in performance was seen for both GA-OLS and GA-MM for chrom.size $\geq 25$. We chose chrom.size $=25$ to be used further. Thus, after optimizing chrom.size, the GA-MM performance was now equal to the GA-OLS performance $\left(\mathrm{R}_{\mathrm{CV}}^{2}=0.87\right)$.

\section{Number of GA runs}

The GA settings for evaluating num.runs are shown in Table 6. Analogously as for max.generations and chrom. size, evaluation was done for both GA-OLS and GA-MM using $3 \times 5$-fold cross-validation (see section III).

In Figure 5 the $R_{C V}^{2}$ performance is shown using all best chromosomes from num.runs in the model averaging (MMI1) (cf. sections III and IV), including the cases where the GA variable selection is done with MM and reestimation of the variables in the MMI is done with OLS and vice versa. A similar $R^{2}$ performance was observed when using GA-OLS or GA-MM for the variable selection. However, a higher $R_{C V}^{2}$ performance was observed when using OLS for estimation of the best chromosome parameters. The $\mathrm{R}_{\mathrm{CV}}^{2}$ performance was stable for num. runs $\geq 10$. When increasing num.runs from 100 to 500 for

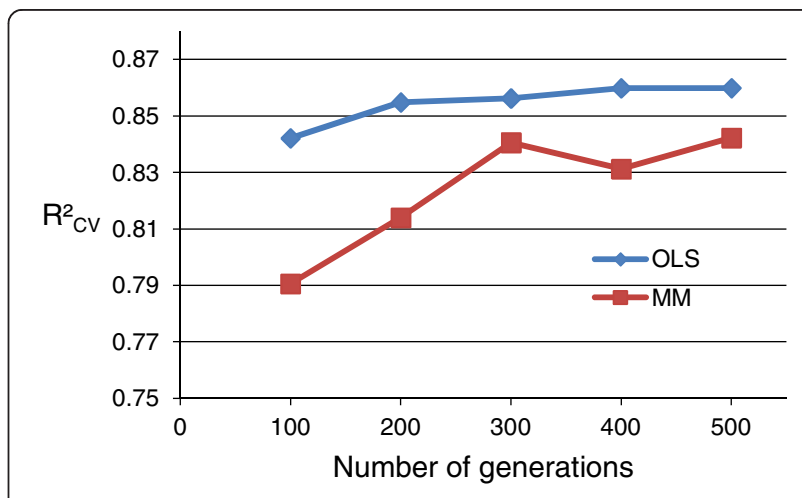

Figure 3 GA parameter: number of generations. $R_{C V}^{2}$ from mean prediction of best chromosomes from 10 runs $(3 \times 5$-fold CV (MMI1). 
Table 5 GA parameter settings to evaluate chrom.size

\begin{tabular}{llll}
\hline GA & CHOSEN & PRE-SET & BEING OPTIMIZED \\
\hline GA-OLS & pop.size $=20$ & num.runs $=10$ & chrom.size $\in$ \\
& & \\
GA-MM & $P e=(1,1,1,0.2)$ & goal.fitness $=1$ & \\
& $P m=0.1$ & \\
& $P C=0.6$ & \\
& tournament.size $=10$ & \\
& max.generations $=400$ & \\
\end{tabular}

GA-OLS variable selection, only a slight increase in $R_{C V}^{2}$ performance was seen.

In Figure 6 the $R_{C V}^{2}$ performance is shown using only the 'best' best chromosome from num.runs for prediction in the cross-validation. Overall, a similar $\mathrm{R}_{\mathrm{CV}}^{2}$ performance was observed when using OLS or MM for estimation of the 'best' best chromosome parameters, and using GA-MM or GA-OLS for variable selection.

In Figure 7, the ' $\mathrm{x} \%$ best' best chromosomes (shown on the $\mathrm{x}$-axis in log scale) were used in the model averaging (MMI1). Evaluation was done for num.runs $=100$ or 500 and num.runs $=50$ or 100 for GA-OLS and GA-MM, respectively. For GA-OLS, the highest $\mathrm{R}_{\mathrm{CV}}^{2}$ was 0.89 and was obtained when including the 'five best' best chromosomes (top $1 \%$ chromosomes with highest $\mathrm{R}^{2}(\mathrm{OLS})$ from num.runs = 500). Also, for GA-MM, including the 'five best' best chromosomes (top 5\% of num.runs $=100$ with highest $R_{M M}^{2}$ ) gave the highest $R^{2}: 0.879$ and 0.885 for MMI-MM and MMI-OLS, respectively. Thus, both for GA-OLS-MMI and GA-MM-MMI inclusion of the 'five best' best chromosomes yielded an improvement in $R_{C V}^{2}$ performance in comparison to using all best chromosomes (Figure 5) or 'the best' best chromosome (Figure 6). As previously noted from Figure 5 , also a better $R_{C V}^{2}$ performance was again found using OLS estimation of parameters than when using MM estimation. For GAOLS num.runs $=100$ was repeated 5 times (splitting the best chromosomes as available from num.runs $=500$ in

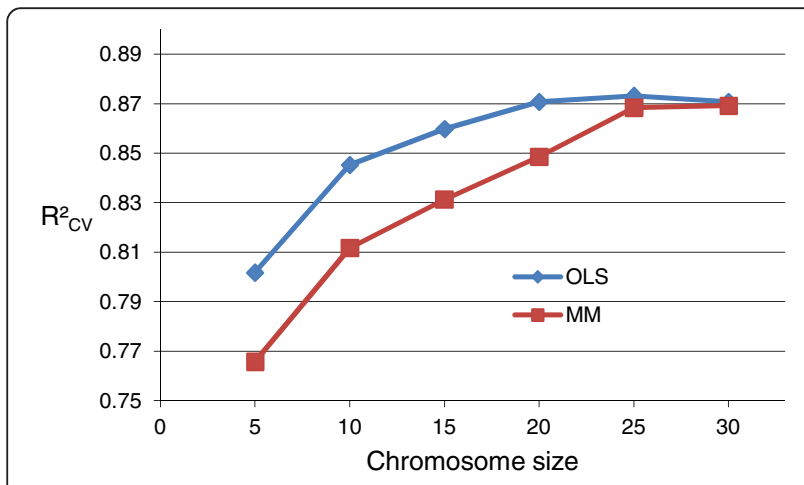

Figure 4 GA parameter: chromosome size. $R_{C V}^{2}$ from mean prediction of best chromosomes from 10 runs $(3 \times 5$-fold CV) (MMI1).
Table 6 GA parameter settings to evaluate num.runs

\begin{tabular}{llll}
\hline GA & CHOSEN & PRE-SET & BEING OPTIMIZED \\
\hline GA-OLS & pop.size $=20$ & goal.fitness $=1$ & num.runs $\in$ \\
GA-MM & chrom.size $=25$ & & $\{1,10,20,50,100,500\}$ \\
& $P e=(1,1,1,0.2)$ & \\
& $P m=0.1$ & \\
& $P C=0.6$ & \\
& tournament.size $=10$ & \\
& max.generations $=400$ & \\
\end{tabular}

five consecutive parts for evaluation using MMI1). The mean curve of these 5 repeats is shown, together with the $95 \%$ confidence interval error bars. The peak of this mean curve is at ' $6 \%$ best' best chromosomes included. The GA-MM curve with MMI-OLS estimation (num. runs $=100)$ is situated within these error bars. Thus, for num.runs $=100$ GA-MM and GA-OLS perform equally well in selecting variables for the model in the crossvalidation. For GA-OLS the $\mathrm{R}_{\mathrm{CV}}^{2}$ performance using the 'five best' best chromosomes using num.runs $=500$ is better than when using num.runs $=100$. Therefore, calculation of the goal fitness for GA-OLS in section VI was done from the num.runs $=500$ best chromosomes. For calculation of the goal fitness for GA-MM, num.runs = 100 was used. Note that, once the goal fitness was calculated, num.runs was set to NA (not applicable). Instead, the model comparison will be based on num.solutions = 100 (number of best chromosomes with $\mathrm{R}^{2}>$ goal.fitness) for both GA-OLS and GA-MM.

\section{Goal fitness}

As derived from Figure 7, for calculating the goal fitness we considered the fitness of the ' 5 best' best chromosomes: this is the top $1 \%$ (of num.runs $=500$ ) for GA-

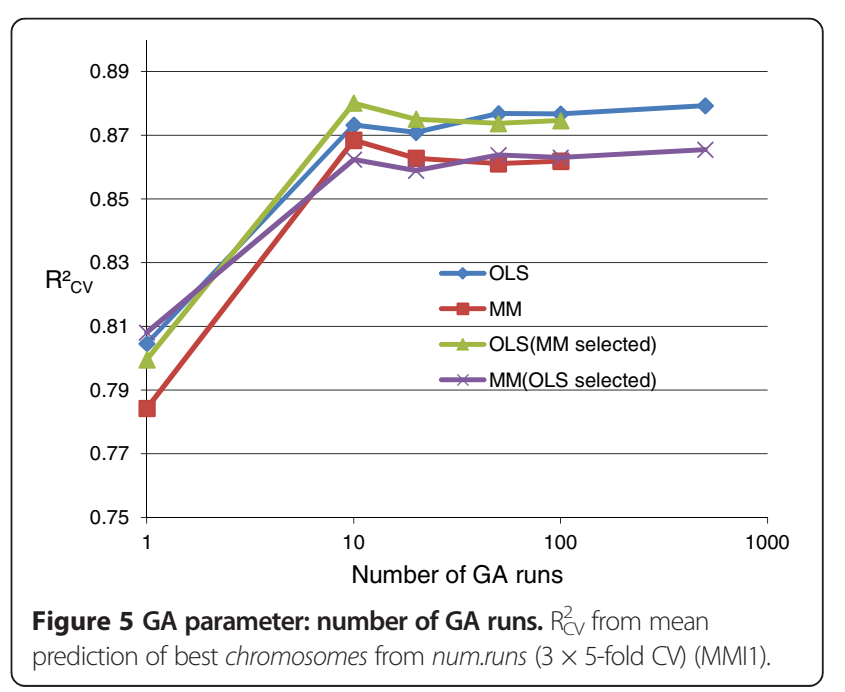




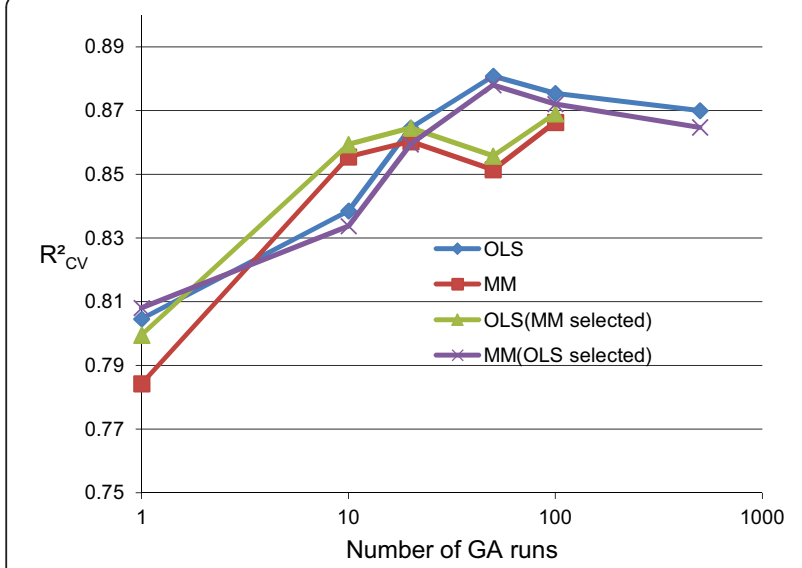

Figure 6 GA parameter: number of GA runs. $R_{C V}^{2}$ from prediction of 'best' best chromosome from num.runs $(3 \times 5$-fold CV).

OLS and the top 5\% (of num.runs = 100) for GA-MM. For each of the $15(3 \times 5) \mathrm{CV}$ training sets we calculated the non-parametric one-sided (1- $p, 1-\alpha)$ tolerance upper limit [26] on the $\mathrm{R}^{2}$ fitness distribution of best chromosomes from num.runs with $p=1 \%$ and $p=5 \%$ for GA-OLS and GA-MM, respectively, and $\alpha=0.05$ (95\% confidence). The interpretation is that with confidence level 1- $\alpha$ not more than $(100 \times p) \%$ of the best chromosomes have $\mathrm{R}^{2}$ fitness values exceeding this limit. To be able to calculate these tolerance limits the requested number of runs was $\left[\frac{\ln (\alpha)}{\ln (1-p)}\right]_{+}$[26]. This requirement was met for num.runs $=100 \geq 59$, and num.runs $=500 \geq 299$ for GA-MM and GA-OLS, respectively. The goal fitness

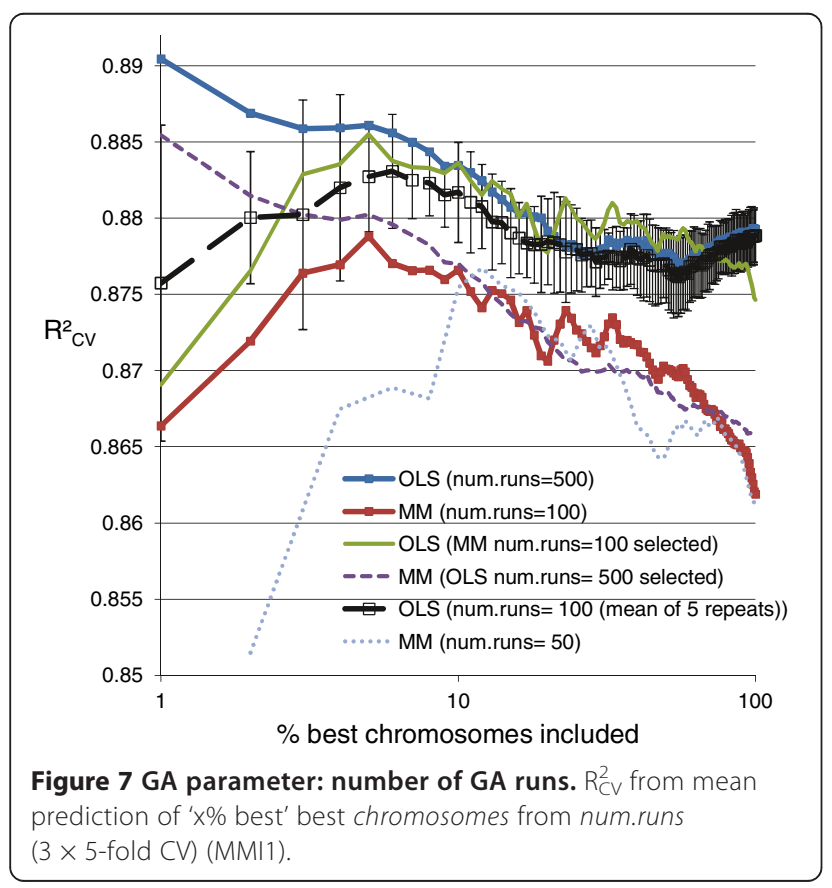

was then calculated as the mean of the $\mathrm{CV}$ tolerance upper limits:

$$
\text { goal.fitness }=\sum_{i=1}^{15} \text { tol.upper }_{i} / 15,
$$

which equals goal.fitness $=0.957$ for GA-OLS and goal . fitness $=0.95$ for GA-MM. For the calculation we used the R package tolerance 0.5.2 [27].

\section{GA-OLS vs. GA-MM: variable selection}

GA-OLS and GA-MM variable selection were performed on the clonal genotype-phenotype database using the GA parameters as specified in the above sections. The percentage of runs that failed reaching the goal.fitness with $\max$. generations $=500$ was $16 \%$ and $23.1 \%$ for GA-OLS and GA-MM, respectively.

Figure 8 shows the relation between the frequency of the variables selected in the GA using OLS and MM. While frequency differences were clearly observed (e.g. for 74M, 151I, 230R, 84L, 140S, 143C, 155S, and 140A), a strong correlation was obtained $\left(R^{2}=0.95\right)$. Eight integrase mutations were selected as variables in all $100 \mathrm{GA}$ solutions for both GA-OLS and GA-MM: 92Q, 97A, 143G, 143R, $148 \mathrm{H}, 148 \mathrm{~K}, 148 \mathrm{R}, 155 \mathrm{H}$. This number would possibly be lower when increasing num.solutions, leading to nonselection for a few GA solutions. This was now already the case with num.runs $=100$ for 66K (always selected by GA-MM, 99/100 selected by GA-OLS) and 121Y (always selected by GA-OLS, 99/100 selected by GA-MM).

\section{GA-OLS and GA-MM variable selection vs. LASSO}

Figure 9 shows the comparison of the GA-OLS and GAMM ranking with the LASSO top 50 ranking of variables, shown on the $\mathrm{x}$-axis. The variables selected are integrase mutations, indicated as primary/secondary/other. Primary and secondary mutations have been associated with RAL resistance in [10]. Note that whereas a single primary mutation causes RAL resistance, the effect on resistance of secondary mutations not in a combination with a primary mutation is minor [5]. In Figure 9, most of the primary and secondary mutations had a high ranking for GAOLS, GA-MM and LASSO. However, some of the 'other' mutations such as 66K, 121Y, $143 \mathrm{G}$ and $155 \mathrm{~S}$ with presence in one or more of the publically available genotypic algorithms: ANRS (http://www.hivfrenchresistance.org), Rega (http://regaweb.med.kuleuven.be), and Stanford (http://hivdb.stanford.edu), had a lower ranking for LASSO. We note that $66 \mathrm{~K}, 121 \mathrm{Y}$ and $155 \mathrm{~S}$ were introduced in the database as site-directed-mutants not in a combination with other mutations [5], and LASSO was less sensitive in selecting these. Another observation was that for LASSO, the secondary mutations $140 \mathrm{~A}$ and $140 \mathrm{~S}$ were ranked higher than the primary mutations. Also, mutations not 


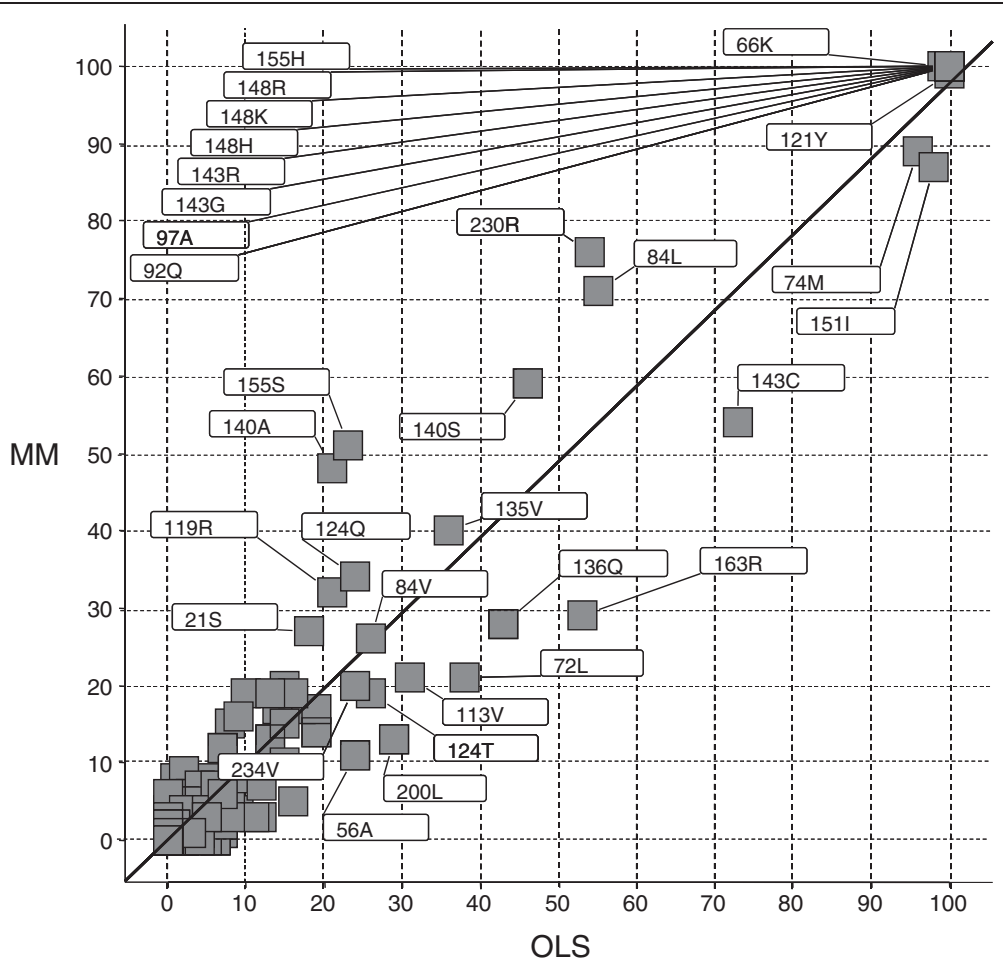

Figure 8 Pearson correlation of GA frequency rankings using MM vs. OLS $\left(\mathbf{R}^{2}=\mathbf{0 . 9 5}\right)$. Mutations with frequency $>20$ for $O L S$ or MM are labeled.

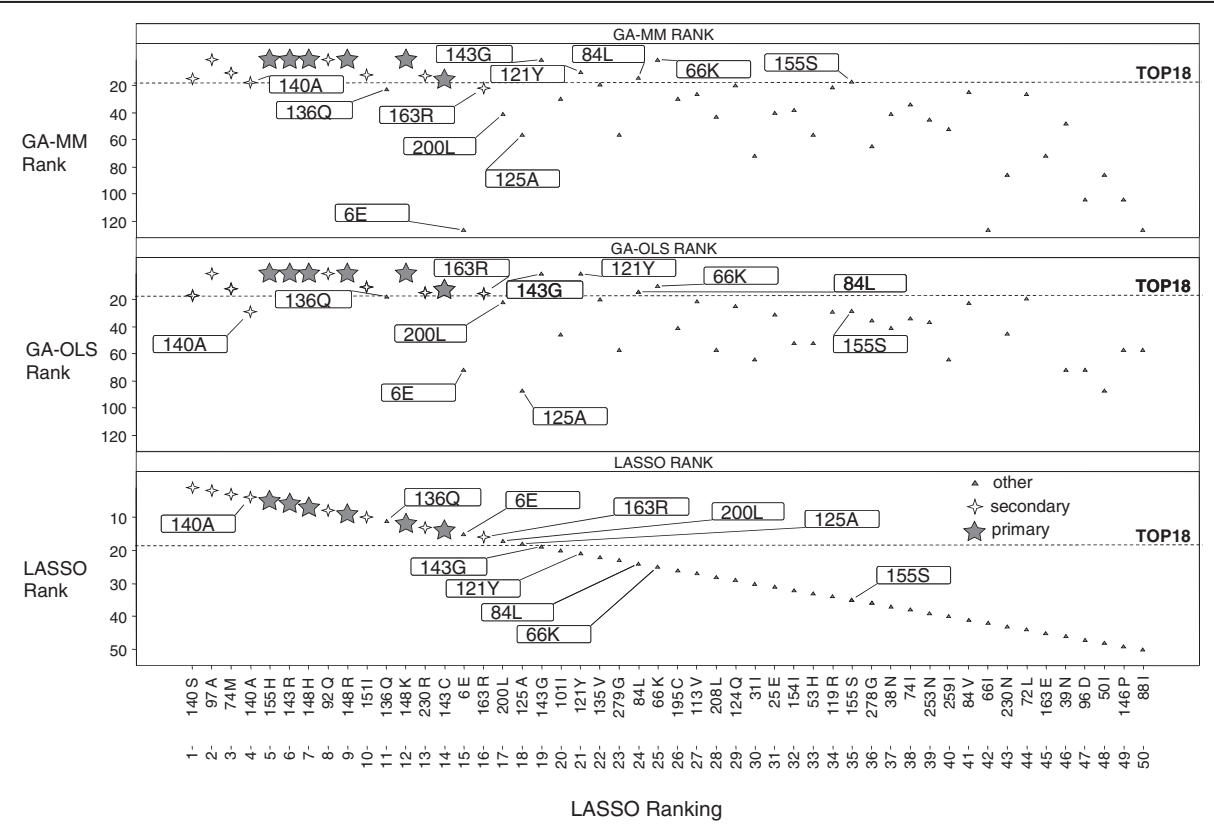

Figure 9 LASSO ranking (50 mutations) compared with GA-MM and GA-OLS. A reference line is shown for the top 18 ranked mutations. Using the RAL product label [10], mutations are indicated as primary/secondary or other. 
Table $7 \mathbf{R}^{2}$ performance on test set 1

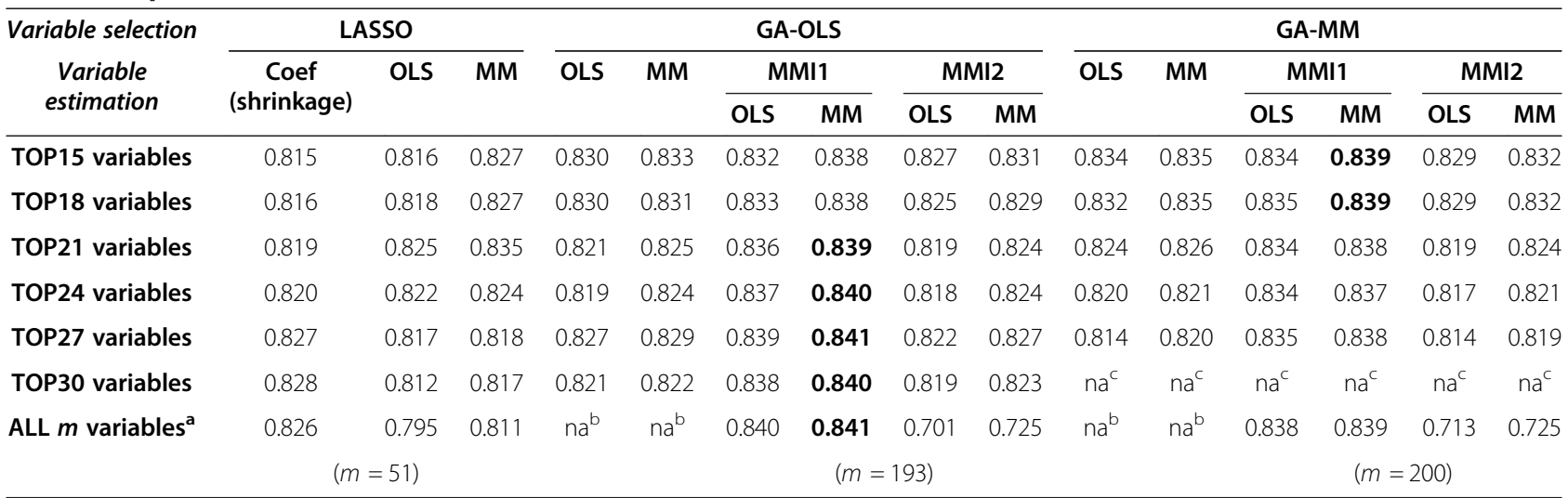

${ }^{a} m$ is the number of variables with presence in the GA solutions or with abs(coef) $>0$ in the LASSO solution path. ${ }^{b}$ not calculated due to singularity.

cno model with exactly 30 mutations.

In bold the highest $\mathrm{R}^{2}$ per row is indicated.

listed by any of the public algorithms such as $6 \mathrm{E}, 125 \mathrm{~A}$, and 200L, had a higher ranking for LASSO in comparison to GA-OLS and GA-MM. Oppositely, one of the "other" integrase mutations ranked higher by GA-OLS and GA-MM, and not listed by any of the public genotypic algorithms was 84L. In [5] we already discussed that its selection may result from a more complex interaction of three secondary mutations with which $84 \mathrm{~L}$ co-occurred in the clones of one clinical isolate.

When we compared the GA-OLS ranking with the GAMM ranking (Figures 8 and 9), a relatively low ranking was seen e.g., for GA-OLS for 140A and 155S, which favours GA-MM for its interpretation.

GA-OLS-MMI vs. GA-MM-MMI vs. LASSO: $\mathrm{R}^{2}$ performance on test set 1 and test set 2

In Tables 7 and 8 are the results of the $\mathrm{R}^{2}$ performance comparison of GA-OLS-MMI, GA-MM-MMI, and LASSO on the two test sets with $n=171$ clinical isolates and $n=67$ site-directed mutants, respectively. Models containing the TOP15-18-21-24-27-30 or ALL variables as selected by LASSO, GA-OLS and GA-MM were considered. Note that as randomness is incorporated in the GA optimization techniques there are more mutations with presence in at least one of the GA solutions, $m=193$ and $m=200$ for GA-OLS and GA-MM respectively, compared to $m=51$ mutations with absolute value of the regression coefficients above zero in the LASSO solution path.

On test set 1, using MM for the variable estimation had a slightly better $\mathrm{R}^{2}$ performance than using OLS, for all models considered. Note that this was not the case in the cross-validation (section $\mathbf{V}$ ) where OLS $R_{C V}^{2}$ performance was higher, possibly due to the inclusion of multiple clinical isolates from the same patient. However, as patient information was not given for the training set, we could not take this into account. For the TOP15/TOP18 models

Table $8 \mathbf{R}^{2}$ performance on test set 2

\begin{tabular}{|c|c|c|c|c|c|c|c|c|c|c|c|c|c|c|c|}
\hline \multirow{3}{*}{$\begin{array}{c}\text { Variable selection } \\
\text { Variable } \\
\text { estimation }\end{array}$} & \multicolumn{3}{|c|}{ LASSO } & \multicolumn{6}{|c|}{ GA-OLS } & \multicolumn{6}{|c|}{ GA-MM } \\
\hline & \multirow{2}{*}{$\begin{array}{c}\text { Coef } \\
\text { (shrinkage) }\end{array}$} & \multirow[t]{2}{*}{ OLS } & \multirow[t]{2}{*}{ MM } & \multirow[t]{2}{*}{ OLS } & \multirow[t]{2}{*}{ MM } & \multicolumn{2}{|c|}{ MMI1 } & \multicolumn{2}{|c|}{ MMI2 } & \multirow[t]{2}{*}{ OLS } & \multirow[t]{2}{*}{ MM } & \multicolumn{2}{|c|}{ MMI1 } & \multicolumn{2}{|c|}{ MMI2 } \\
\hline & & & & & & OLS & $\mathrm{MM}$ & OLS & $\mathrm{MM}$ & & & OLS & $\overline{M M}$ & OLS & $\mathrm{MM}$ \\
\hline TOP15 variables & 0.667 & 0.734 & 0.712 & 0.707 & 0.707 & 0.708 & 0.708 & 0.709 & 0.710 & 0.709 & 0.702 & 0.705 & 0.696 & 0.706 & 0.698 \\
\hline TOP18 variables & 0.690 & 0.731 & 0.713 & 0.721 & 0.718 & 0.716 & 0.714 & 0.722 & 0.719 & 0.768 & 0.770 & 0.742 & 0.742 & 0.747 & 0.750 \\
\hline TOP21 variables & 0.742 & 0.760 & 0.765 & 0.736 & 0.730 & 0.722 & 0.717 & 0.732 & 0.726 & 0.777 & 0.775 & 0.746 & 0.744 & 0.751 & 0.752 \\
\hline TOP24 variables & 0.745 & 0.771 & 0.768 & 0.732 & 0.728 & 0.720 & 0.716 & 0.727 & 0.723 & 0.762 & 0.761 & 0.743 & 0.740 & 0.748 & 0.749 \\
\hline TOP27 variables & 0.767 & 0.788 & 0.788 & 0.721 & 0.725 & 0.720 & 0.717 & 0.732 & 0.726 & 0.770 & 0.768 & 0.744 & 0.741 & 0.758 & 0.755 \\
\hline TOP30 variables & 0.777 & 0.789 & 0.787 & 0.768 & 0.772 & 0.731 & 0.729 & 0.747 & 0.743 & $n a^{c}$ & nac & $n a^{c}$ & nac & nac & $n a^{c}$ \\
\hline \multirow[t]{2}{*}{ ALL $m$ variables ${ }^{a}$} & 0.787 & 0.770 & 0.776 & $n a^{b}$ & $n a^{b}$ & 0.733 & 0.729 & 0.741 & 0.733 & $n a^{b}$ & $n a^{b}$ & 0.747 & 0.745 & 0.754 & 0.749 \\
\hline & \multicolumn{3}{|c|}{$(m=51)$} & \multicolumn{6}{|c|}{$(m=193)$} & & & \multicolumn{4}{|c|}{$(m=200)$} \\
\hline
\end{tabular}

${ }^{a} m$ is the number of variables with presence in the GA solutions or with abs(coef) $>0$ in the LASSO solution path. ${ }^{b}$ not calculated due to singularity.

c no model with exactly 30 mutations.

In bold the highest $\mathrm{R}^{2}$ per row is indicated. 
containing the smallest number of variables, the best performance was seen for GA-MM-MMI1 $\left(R^{2}=0.839\right)$. For the TOP21- > ALL models with more variables considered, the best performance was seen for GA-OLS-MMI1 $\left(\mathrm{R}^{2}=0.839-0.841\right)$. When estimating ALL GA-OLS/ GA-MM variables, the worst performance was seen for MMI2 $\left(R^{2}=0.701-0.725\right)$ where noise variables were clearly overweighted. For LASSO, the best $\mathrm{R}^{2}$ performance on test set 1 was obtained using MM for the variable estimation for the TOP15- > TOP24 selection of variables $\left(\mathrm{R}^{2}=0.824-0.835\right)$. For LASSO TOP27- > ALL, the best $\mathrm{R}^{2}$ performance was obtained using the LASSO shrinkage coefficients $\left(R^{2}=0.826-0.828\right)$.

On test set 2, for the sparse models the best performance was observed for LASSO-OLS TOP15 $\left(R^{2}=0.734\right)$, GA-MM-MM TOP18 $\left(\mathrm{R}^{2}=0.770\right)$, and GA-MM-OLS TOP21 $\left(R^{2}=0.777\right)$. For the TOP21- > ALL models, the best performance was seen for LASSO $\left(R^{2}=0.771-0.789\right)$. In contrast to the results for test set 1 , the MMI2 $R^{2}$ performance was now found to be higher than for MMI1, for the GA-OLS/MM models. The reason is that while test set 1 consisted of clinical samples, with $82.5 \%$ not containing any of the primary RAL resistance mutations [5], test set 2 consisted of site-directed mutants containing most of the known resistance patterns but lacking any noise variables as found in clinical samples. Nevertheless, on test set 2 , the GA-MM $R^{2}$ values were found to be better than for GA-OLS, confirming that a better selection of variables as made by GA-MM (cf. the above two sections) led to a better performance on unseen data.

Therefore, on the example training set in this article we would favour the GA-MM-MMI TOP18 model. Based on the performance on test set 2, for the MMI variable estimation re-fitting using MM may be preferred over MMI1-MM.

\section{Conclusions}

In this article, we extended our GA variable selection methodology to mixed models which account for clustering in the data. Using cross-validation, we optimized the GA parameter settings taking also computational efficiency into account. For the worked-out example, all settings could be taken equal for GA-OLS and GA-MM, with exception of goal.fitness for which we used a marginal $\mathrm{R}^{2}$ definition. The model parameters for prediction could then be estimated using MMI-MM (REML) on the GA solutions obtained from $100 \mathrm{GA}$ runs. When testing LASSO, GA-OLS and GA-MM on two unseen data sets, all methods had good performance. When imposing a parsimony restriction for better interpretability of the model, the GA-MM-MMI TOP18 model had better predictive accuracy $\left(\mathrm{R}^{2}\right)$ than GA-OLS and LASSO.

In summary, we belief that GA-MM-MMI is a direct approach to derive a sparse and interpretable model for making predictions with good accuracy on small data sets with clustered observations and a large number of candidate variables, where chance of overfitting with standard regression techniques is high.

\section{Availability and requirements}

Project name: GA-MM-MMI.

Project home page: http://sourceforge.net/projects/ ga-mm-mmi.

Operating system: Platform independent.

Programming language: $R \geq 2.15 .2$, perl, MATLAB.

Other requirements: requires galgo 1.0.11 [23].

License: GNU GPL.

Any restrictions to use by non-academics: none.

\section{Additional files}

\section{Additional file 1: GA parameter settings and computational}

efficiency. Computational efficiency is taken into account by setting the GA parameters one by one: I and II: Pm and PC, II tournament.size, III max.generations, IV chrom.size, $\mathbf{V}$ num.runs (needed until goal.fitness is set in VI). Pre-set values are used for the parameters to be optimized later in the process. For deriving the GA parameters in III - > V we used $3 \times 5$-fold cross-validation.

Additional file 2: $\mathbf{R}$ code. GA-MM-MMI source code in $\mathrm{R}$ programming language ( $\mathrm{R} \geq 2.15 .2$ from http://cran.r-project.org).

\section{Competing interests}

The authors declare that they have no competing interests

\section{Authors' contributions}

KVdB developed the GA-MM-MMI approach, performed all analyses and wrote the manuscript. GV conceived of the study, assisted in its design, and revised the manuscript. HvV assisted in study design. All authors read and approved the final manuscript.

\section{Acknowledgements}

The authors would like to thank the anonymous reviewers for their constructive comments to improve the manuscript. Financial support from the IAP research network \#P7/06 of the Belgian Government (Belgian Science Policy) is gratefully acknowledged.

\section{Author details}

${ }^{1}$ Janssen Infectious Diseases-Diagnostics BVBA, B-2340 Beerse, Belgium. ${ }^{2}$ Interuniversity Institute for Biostatistics and statistical Bioinformatics, Katholieke Universiteit Leuven, B-3000 Leuven, Belgium. ${ }^{3}$ Interuniversity Institute for Biostatistics and statistical Bioinformatics, Universiteit Hasselt, B-3590 Diepenbeek, Belgium.

Received: 11 October 2013 Accepted: 21 March 2014

Published: 27 March 2014

\section{References}

1. Orelien JG, Edwards $\sqcup$ : Fixed-effect variable selection in linear mixed models using $R^{2}$ statistics. Comput Stat Data An 2008, 52:1896-1907.

2. Schelldorfer J, Bühlmann P, Van de Geer S: Estimation for high-dimensional linear mixed-effects models using I1-penalization. Scand J Stat 2011, 38:197-214.

3. Taylor JD, Verbyla AP, Cavanagh C, Newberry M: Variable selection in linear mixed models using an extended class of penalties. Aust N Z J Stat 2012, 54:427-449.

4. Hajjem A, Bellavance F, Larocque D: Mixed-effects random forest for clustered data. J Stat Comput Simul 2012 [http://dx.doi.org/10.1080/ 00949655.2012.741599]. 
5. Van der Borght $\mathrm{K}$, Verheyen A, Feyaerts M, Van Wesenbeeck L, Verlinden $\mathrm{Y}$, Van Craenenbroeck E, van Vlijmen H: Quantitative prediction of integrase inhibitor resistance from genotype through consensus linear regression modeling. Virol J 2013, 10:8,

6. Verbeke G, Molenberghs G: Linear Mixed Models for Longitudinal Data. New York: Springer; 2000.

7. Zuur AF, leno EN, Walker N, Saveliev AA, Smith GM: Mixed Effects Models and Extensions in Ecology with R. New York: Springer; 2009.

8. Kutner MH, Nachtsheim CJ, Neter J, Li W: Applied Linear Statistical Models. New York: McGraw-Hill; 2004.

9. Tibshirani R: Regression Shrinkage and Selection via the Lasso. J Royal Stat Soc B 1996, 58:267-288.

10. FDA: Isentress (raltegravir) drug label. 2009 [http://www.accessdata.fda. gov/drugsatfda_docs/label/2009/022145s004lbl.pdf].

11. Affenzeller M, Winkler S, Wagner S, Beham A: Genetic Algorithms and Genetic Programming - Modern Concepts and Practical Applications. Boca Raton: CRC Press; 2009.

12. Butz MV: Rule-Based Evolutionary Online Learning Systems - A Principled Approach to LCS Analysis and Design. Berlin: Springer; 2006.

13. Hopgood AA: Intelligent Systems for Engineers and Scientists. Boca Raton: CRC Press; 2001

14. Sivanandam SN, Deepa SN: Introduction to Genetic Algorithms. Heidelberg: Springer; 2008.

15. Michalewicz Z: Genetic Algorithms + Data Structures = Evolution Programs New York: Springer; 1996

16. Edwards $L$, Muller $K E$, Wolfinger RD, Quaqish $B F$, Schabenberger $O$ : An $R^{2}$ statistic for fixed effects in the linear mixed model. Stat Med 2008, 27:6137-6157.

17. Kramer $M: R^{2}$ statistics for mixed models. In Proceedings of the $17^{\text {th }}$ annual Kansas State University Conference on Applied Statistics in Agriculture: 25-27 April 2005. Manhattan, Kansas. Kansas State University; 2005:148-160.

18. Nakagawa S, Schielzeth $H$ : A general and simple method for obtaining $R^{2}$ from generalized linear mixed-effects models. Methods Ecol Evol 2013, 4:133-142.

19. Flack VF, Chang PC: Frequency of selecting noise variables in subset regression analysis: a simulation study. Am Stat 1987, 41:84-86.

20. Lukacs PM, Burnham KP, Anderson DR: Model selection bias and Freedman's paradox. Ann Inst Stat Math 2010, 62:117-125.

21. Friedman J, Hastie T, Tibshirani R: Regularization paths for generalized linear models via coordinate descent. J Stat Softw 2010, 33:1. R package version 1.9-3. [http://CRAN.R-project.org/package $=$ glmnet].

22. Belloni $A$, Chernozhukov V: Least squares after model selection in high-dimensional sparse models. Bernoulli 2013, 19:521-547.

23. Trevino V, Falciani F: GALGO: an R package for multivariate variable selection using genetic algorithms. Bioinformatics 2006, 22:1154-1156. R package version 1.0.11. [http://biptemp.bham.ac.uk/vivo/galgo/ AppNotesPaper.htm].

24. Tenorio F: gaoptim: Genetic Algorithm optimization for real-valued problems; 2013. $R$ package version 1.0. [http://CRAN.R-project.org/package=gaoptim].

25. Miller BL, Goldberg DE: Genetic algorithms, tournament selection, and the effects of noise. Complex Systems 1995, 9:193-212.

26. Krishnamoorthy K, Mathew T: Statistical Tolerance Regions: Theory, Applications, and Computation. Hoboken, NJ: John Wiley \& Sons; 2009.

27. Young DS: An R package for estimating tolerance intervals. J Stat Softw 2010, 36:5. R package version 0.5.2. [http://CRAN.R-project.org/package=tolerance].

\section{doi:10.1186/1471-2105-15-88}

Cite this article as: Van der Borght et al:: Multi-model inference using mixed effects from a linear regression based genetic algorithm. BMC Bioinformatics 2014 15:88.

\section{Submit your next manuscript to BioMed Central and take full advantage of:}

- Convenient online submission

- Thorough peer review

- No space constraints or color figure charges

- Immediate publication on acceptance

- Inclusion in PubMed, CAS, Scopus and Google Scholar

- Research which is freely available for redistribution

Submit your manuscript at www.biomedcentral.com/submit
Ciomed Central 